Mazur Karol, Lewicki Marcin, Mazur Dominika, Smoleń Agata. Efficacy and safety of ashwagandha root extract in the treatment of insomnia, anxiety and reducing stress - literature review. Journal of Education, Health and Sport. 2021;11(12):197-202. eISSN 23918306. DOI http://dx.doi.org/10.12775/JEHS.2021.11.12.013

https://apcz.umk.pl/JEHS/article/view/JEHS.2021.11.12.013

https://zenodo.org/record/5783850

The journal has had 40 points in Ministry of Education and Science of Poland parametric evaluation. Annex to the announcement of the Minister of Education and Science of December 1, 2021. No. 32343. Has a Journal's Unique Identifier: 201159. Scientific disciplines assigned: Physical Culture Sciences (Field of Medical sciences and health sciences); Health Sciences (Field of Medical Sciences and Health Sciences).

Punkty Ministerialne z 2019 - aktualny rok 40 punktów. Zalącznik do komunikatu Ministra Edukacji i Nauki z dnia 1 grudnia 2021 r. Lp. 32343. Posiada Unikatowy Identyfikator Czasopisma: 201159. Przypisane dyscypliny naukowe:Nauki o kulturze fizycznej (Dziedzina nauk medycznych i nauk o zdrowiu); Nauki o zdrowiu (Dziedzina nauk medycznych i nauk o zdrowiu).

(ㅇ) The Authors 2021;

This article is published with open access at Licensee Open Journal Systems of Nicolaus Copernicus University in Torun, Polan

Open Access. This article is distributed under the terms of the Creative Commons Attribution Noncommercial License which permits any noncommercial use, distribution, and reproduction in any medium,

provided the original author (s) and source are credited. This is an open access article licensed under the terms of the Creative Commons Attribution Non commercial license Share alike.

(http://creativecommons.org/licenses/by-nc-sa/4.0/) which permits unrestricted, non commercial use, distribution and reproduction in any medium, provided the work is properly cited.

The authors declare that there is no conflict of interests regarding the publication of this paper.

Received: 25.11.2021. Revised: 30.11.2021. Accepted: 15.12.2021.

\title{
Efficacy and safety of ashwagandha root extract in the treatment of insomnia, anxiety and reducing stress - literature review
}

\section{Karol Mazur ${ }^{1}$, Marcin Lewicki ${ }^{2}$, Dominika Mazur ${ }^{3}$, Agata Smoleń ${ }^{4}$}

${ }^{1}$ Doctoral School, Medical University of Lublin, ul. Chodźki 7, 20-093 Lublin, Poland; mazurkaro179@gmail.com; https://orcid.org/0000-0003-3459-2588;

${ }^{2}$ Department of Epidemiology and Clinical Research Methodology of the Medical University of Lublin, ul. Radziwiłłowska 11, 20-080 Lublin, Poland; lewicki-marcin@wp.pl; https://orcid.org/0000-0003-1906-9326;

${ }^{3}$ Department of Obstetrics and Pathology of Pregnancy, Independent Public University Hospital No. 1 in Lublin, ul. Staszica 16, 20-081 Lublin, Poland; dominika.hul20@gmail.com; https://orcid.org/0000-0002-4689-0462;

${ }^{4}$ Department of Epidemiology and Clinical Research Methodology of the Medical University of Lublin, ul. Radziwiłłowska 11, 20-080 Lublin, Poland; agatasmolen@umlub.pl; https://orcid.org/0000-0003-0764-6667;

\section{Summary:}

Insomnia, anxiety and stress are common complaints of people living today. Together, these factors can significantly reduce the quality of people's lives and disrupt relationships. Insomnia is a health problem connected with the difficulty falling asleep, waking early, waking during sleep, or poor quality of sleep, the consequences of which during the day are a feeling of being fatigue, reduced well-being, irritability, impaired concentration and learning ability. Anxiety is an emotional state related with anticipation of danger coming from the outside or coming from inside the organism, manifesting itself as feeling of uneasiness, 
tension, embarrassment, threat. Stress is a disturbance of body homeostasis caused by a physical or psychological factor.

The aim of this study was to assess the efficacy and safety of ashwagandha root extract in the treatment of insomnia, anxiety and reducing stress. Our study material consisted of publications, which were found in PubMed, ResearchGate and Google Scholar databases. In order to find the proper publications, the search has been conducted with the use of a combination of key words like: "ashawagandha", "insomnia", "anxiety", "stress". The first step was to find proper publications from the last 5 years. The second step was to carry out an overview of the found publications.

Currently a lot of researches is being conducted to find natural substances that are just as effective as pharmacotherapy in the treatment of insomnia, anxiety and reducing stress, but without the side effects. Some researchers have focused their attention to the ashwagandha root extract. Results of mentioned studies proved the efficacy and safety of ashwagandha root extract. However, further research is needed to estabilish the role of ashwagandha root extract in the treatment of insomnia, anxiety and reducing stress.

Key words: ashawagandha, insomnia, anxiety, stress

\section{INTRODUCTION AND PURPOSE}

Insomnia, anxiety and stress are common complaints of people living today. Together, these factors can significantly reduce the quality of people's lives and disrupt relationships. Insomnia is a health problem connected with the difficulty falling asleep, waking early, waking during sleep, or poor quality of sleep, the consequences of which during the day are a feeling of being fatigue, reduced well-being, irritability, impaired concentration and learning ability. The main causes of insomnia are: taking naps during the day, taking stimulants such as coffee, drugs, alcohol, lack of physical activity during the day, eating fatty food before bedtime, lack of peace, quiet and the presence of electronic devices in the bedroom, reading books or watching television in bed [1]. Anxiety is an emotional state related with anticipation of danger coming from the outside or coming from inside the organism, manifesting itself as feeling of uneasiness, tension, embarrassment, threat. There is a strong relationship between anxiety and stress. Stress is a disturbance of body homeostasis caused by a physical or psychological factor. The two main biological systems involved in the stress 
response are the sympathetic nervous system and the hypothalamic-pituitary-adrenal axis. The sympathetic nervous system is activated in the first moments after a stressor and is responsible for the so-called fight or flight response. It stimulates the adrenal glands to secrete adrenaline and noradrenaline and produces effects such as dilation of the pupils, acceleration of the pulse and respiration, and increased heart rate [2].

Most of the drugs that are used for the treatment of this disorders cause many adverse effects. Currently a lot of researches is being conducted to find natural substances that are just as effective as pharmacotherapy, but without the side effects. Some researchers have focused their attention to the ashwagandha root extract [3]. Ashawagandha (Withania somnifera L.) commonly known as Indian ginseng, winter cherry or gooseberry, is a xerophytic plant belonging to the Solanaceae family Its natural habitat is Southeast Asia. This plant was used in Ayurveda as a medicinal herb for thousands of years. Ashwagandha shows variability in phytochemical composition depending on the geographical latitude in which it occurs. Most of its chemicals components are alkaloids and steroidal lactones [4]. Many researches demonstrated the efficacy and safety of ashwagandha root extract in the treatment of insomnia, anxiety and reducing stress. Few trails also showed the neuroprotective, cardioprotective, immunomodulatory, anti-diabetic and anti-inflammatory properties of this substance.

The aim of this study was to assess the efficacy and safety of ashwagandha root extract in the treatment of insomnia, anxiety and reducing stress. Our study material consisted of publications, which were found in PubMed, ResearchGate and Google Scholar databases. In order to find the proper publications, the search has been conducted with the use of a combination of key words like: "ashawagandha", "insomnia", "anxiety", "stress". The first step was to find proper publications from the last 5 years. The second step was to carry out an overview of the found publications.

\section{LITERATURE REVIEW}

Salve et al. in their prospective, randomized, double-blind, placebo-controlled study investigated the adaptogenic and anxiolytic effects of ashwagandha root extract. 60 participants were asked to take two capsules a day of ashwagandha extract $125 \mathrm{mg}, 300 \mathrm{mg}$ or placebo for 8 weeks. Screening parameters of the partcipants were assessed using Perceived Stress Scale, Hamilton-Anxiety Scale and serum cortisol measurement at the beginning, in the middle and at the end of the study. The results of this study showed significant reduction in Perceived Stress Scale scores in the ashwagandha $250 \mathrm{mg}$ /day group $(\mathrm{p}<0.05)$ and in the 
ashwagandha $600 \mathrm{mg}$ /day group ( $\mathrm{p}<0.0001)$, in comparison with the placebo group. The scores of the Hamilton Anxiety Rating Scale were not significantly lower in the ashwagandha $250 \mathrm{mg} /$ day group, but were statistically significantly lower $(\mathrm{p}<0.05)$ in the ashwagandha 600 $\mathrm{mg}$ /day group. The decrease cortisol was statistically significantly greater both in the ashwagandha $250 \mathrm{mg}$ /day group $(\mathrm{p}<0.05)$ and in the ashwagandha $600 \mathrm{mg} /$ day group $(\mathrm{p}<$ $0.0001)$, than in the placebo group. The increase in the sleep quality score, was statistically significantly greater both in the ashwagandha $250 \mathrm{mg} /$ day group $(\mathrm{p}<0.05)$ and in the ashwagandha $600 \mathrm{mg} /$ day group $(\mathrm{p}<0.0001)$ in comparison with the placebo group. This indicates that taking ashwagandha root extract $250 \mathrm{mg}$ /day is not as effective as taking ashwagandha root extract $600 \mathrm{mg} /$ day in the treatment of insomnia, anxiety and reducing stress. In this study no serious adverse effects were reported by the participants [5].

Langade et al. in their double-blind, randomized, placebo-controlled study tested the efficacy and safety of ashwagandha root extract in the treatment of insomnia and anxiety. 60 participants were randomized to receive two capsules a day $300 \mathrm{mg}$ ashwagandha root extract or placebo for 10 weeks. Screening parameters of the partcipants were assessed using sleep actigraphy, Pittsburgh Sleep Quality Index and Hamilton Anxiety Rating Scale at the beginning, in the middle and at the end of the study. The results of this study showed statistically significant improvement of the sleep parameters assessed using the actigraphy: Sleep Onset Latency, Total Sleep Time, Wake After Sleep Onset, Total Time in Bed and Sleep Efficiency in the ashwagandha $600 \mathrm{mg}$ /day group, in comparison with the placebo group. The study also noted significant reduction in Pittsburgh Sleep Quality Index scores $(p<0.0001)$ and Hamilton Anxiety Rating Scale scores $(p=0.002)$ in the ashwagandha 600 $\mathrm{mg}$ /day group, in comparison with the placebo group. In this study no serious adverse effects were reported by the participants. Outcomes of this research proved the safety and efficacy of ashwagandha root extract in the treatment of insomnia and anxiety [6].

Chandrasekhar et al. in their prospective, double-blind, randomized, placebo-controlled trial also investigated the ashwagandha root extract in reducing stress and anxiety. 64 participants were randomized to receive two capsules a day $300 \mathrm{mg}$ ashwagandha root extract or placebo for 60 days. Screening parameters of the partcipants were assessed using Perceived Stress Scale, General Health Questionnaire-28, Depression Anxiety Stress Scale and serum cortisol measurement at the beginning and at the end of the study. The results of this study showed significant reduction in Perceived Stress Scale scores $(p<0.0001)$, General Health Questionnaire-28 scores $(\mathrm{p}<0.0001)$ and Depression Anxiety Stress Scale scores $(\mathrm{p}<0.0001)$, 
in the ashwagandha $600 \mathrm{mg} /$ day group, in comparison with the placebo group. The reduction of serum cortisol levels statistically significantly greater in the ashwagandha $600 \mathrm{mg} /$ day group ( $\mathrm{p}=0.002$ ). In this study no serious adverse effects were reported by the participants. Findings of these research suggest that ashwagandha root extract can be safely used as an adaptogen to the stress and thereby improve quality of life [7].

Lopresti et al. in their randomized, double-blind, placebo-controlled study checked the role of ashwagandha root extract in reduction of anxiety and stress. 60 participants were randomized to take either one capsules a day $240 \mathrm{mg}$ ashwagandha root extract or placebo for 60 days. Screening parameters of the partcipants were assessed using the Hamilton Anxiety Rating Scale, Depression, Anxiety, and Stress Scale -21 (DASS-21), and serum levels of cortisol, dehydroepiandrosterone-sulphate, and testosterone. The results of this study showed significant reduction in Hamilton Anxiety Rating Scale scores in the ashwagandha 240 $\mathrm{mg}$ /day group $(\mathrm{p}<0.0001)$, in comparison with the placebo group. Reduction in the Depression, Anxiety, and Stress Scale -21 scores was near-significant in the ashwagandha 240 $\mathrm{mg}$ /day group, in comparison with the placebo group $(\mathrm{p}=0.096)$. Cortisol $(\mathrm{p}<0.001)$ and dehydroepiandrosterone-sulphate $(p=0.004)$ serum levels were statistically significantly lower in the ashwagandha $240 \mathrm{mg}$ /day group, in comparison with the placebo group. Testosterone serum level change was not statistically significant in the ashwagandha $240 \mathrm{mg} /$ day group, in comparison with the placebo group $(\mathrm{p}=0.158)$. In this study no serious adverse effects were reported by the participants [8].

\section{CONCLUSIONS}

1. Currently researches researchers have focused their attention to find natural substances that are just as effective as pharmacotherapy in the treatment of insomnia, anxiety and reducing stress, but without the side effects.

2. Results of mentioned studies proved the efficacy and safety of ashwagandha root extract.

3. Further trails, especially with a larger cohort and in diverse populations, are needed to estabilish the role of ashwagandha root extract in the treatment of insomnia, anxiety and reducing stress.

4. There is also a need for further research to prove the effect of ashwagandha root extract in improving memory, cognitive functions and cardiorespiratory endurance. 


\section{LIST OF REFERENCES:}

1. Roth T. Insomnia: definition, prevalence, etiology, and consequences. J Clin Sleep Med. 2007 Aug 15;3(5 Suppl):S7-10.

2. Cohen S, Janicki-Deverts D, Miller GE. Psychological stress and disease. JAMA. 2007;298(14):1685-1687.

3. Provino R. The role of adaptogens in stress management. Aust J Med Herbal. 2010;22:419.

4. An overview on Ashwagandha: a rasayana (rejuvenator) of Ayurveda. Singh N, Bhalla M, de Jager P, Gilca M. Afr J Tradit Complement Altern Med. 2011;8:208-213.

5. Salve J, Pate S, Debnath K, Langade D. Adaptogenic and Anxiolytic Effects of Ashwagandha Root Extract in Healthy Adults: A Double-blind, Randomized, Placebocontrolled Clinical Study. Cureus. 2019 Dec 25;11(12):e6466.

6. Langade D, Kanchi S, Salve J, Debnath K, Ambegaokar D. Efficacy and Safety of Ashwagandha (Withania somnifera) Root Extract in Insomnia and Anxiety: A Doubleblind, Randomized, Placebo-controlled Study. Cureus. 2019 Sep 28;11(9):e5797.

7. Chandrasekhar K, Kapoor J, Anishetty S. A prospective, randomized double-blind, placebo-controlled study of safety and efficacy of a high-concentration full-spectrum extract of ashwagandha root in reducing stress and anxiety in adults. Indian J Psychol Med. 2012 Jul;34(3):255-62.

8. Lopresti AL, Smith SJ, Malvi H, Kodgule R. An investigation into the stress-relieving and pharmacological actions of an ashwagandha (Withania somnifera) extract: A randomized, double-blind, placebo-controlled study. Medicine (Baltimore). 2019 Sep;98(37):e17186. 